\title{
RECONSTRUCCIÓN DEL MODO DE VIDA DE INDIVIDUOS DEL ARCAICO DE LA COSTA ARREICA DEL NORTE DE CHILE: UNA APROXIMACIÓN BIOARQUEOLÓGICA DESDE EL SITIO COPACA 1
}

\author{
A RECONSTRUCTION OF ARCHAIC FISHERMEN'S LIFESTYLE \\ IN NORTHERN CHILE'S HIPER ARID COAST: \\ A BIOARCHAEOLOGICAL PRESPECTIVE FROM COPACA 1
}

\author{
Pedro Andrade ${ }^{1}$ Victoria Castro $^{2,3}$ y Carlos Aldunate Cl $^{4}$
}

\begin{abstract}
Este trabajo da a conocer datos bioantropológicos inéditos obtenidos de una muestra discreta no disturbada proveniente de excavaciones estratigráficas practicadas en un sitio de tiempos arcaicos, ubicados en la costa arreica de Chile, de los cuales se obtuvieron fechados radiocarbónicos como isótopos estables $(\delta 15 \mathrm{~N}, \delta 13 \mathrm{C} \mathrm{col})$, permitiendo situarlos dentro de la secuencia zonal. A partir de la coherencia de estos resultados se establece que el modo de vida de estos individuos habría estado fuertemente ligado con la explotación de las distintas dimensiones productivas que presenta el ambiente marino, dejando huellas importantes en su registro óseo y dental.
\end{abstract}

Palabras claves: desierto de Atacama, pescadores, cazadores y recolectores, período Arcaico, modos de vida, bioarqueología.

\begin{abstract}
The aim of this paper is to study human biological remains from stratigraphic excavations of the Archaic desertic coastal site of Copaca 1, Northern Chile. The discussion of new chronological, biological and stable isotopes data allows a new approach to the societies that lived in this extremely arid zone of the Atacama Desert. Results obtained in osteological evidence is described, suggesting way of life highly linked with the exploitation of different productive dimensions of the maritime environment through activities patterns that left important traces in their osteological and dental record. coastal diet.

Key words: Desert coast of Northern Chile, Archaic Period, Archaic fishermen-hunter-gatherers, way of life, early
\end{abstract}

Los estudios bioarqueológicos en la costa arreica del norte de Chile han sido escasos, concentrándose primordialmente en el sitio Caleta Huelén 42, enfocados en conocer los ancestros de estos individuos, con el fin de ligarlos con otras poblaciones (Cocilovo et al. 2005). El análisis de los individuos del sitio La Chimba 13, que constituye una muestra más bien escasa, se ha orientado a conocer el patrón funerario (Costa-Junquiera 2001), situación que se replica en el caso de la investigación realizada en el sitio Los Bronces (Llagostera y Llagostera 2010). Lo anterior no se corresponde con el hecho de que existan pocos restos óseos disponibles en la zona, sino que se debe a factores que tienen que ver con la conservación de los mismos, especialmente debido al huaqueo permanente de los cementerios durante años (Castro et al. 2010; Llagostera y Cruz 2010).

Este panorama muestra un vacío en cuanto al conocimiento antropológico físico de esta zona, cuando se le compara con las áreas costeras aledañas de la costa de valles occidentales y del norte semiárido. No obstante lo anterior, las investigaciones sistemáticas en la zona (Castelleti 2007; Castro et al. 2012; Salazar et al. 2011) han generado conocimiento a partir de la excavación de cementerios y áreas saqueadas permitiendo construir una base de datos para acercarnos al conocimiento bioarqueológico de las poblaciones de la costa arreica, principalmente en los períodos Arcaico y

\footnotetext{
Universidad de Concepción, Concepción, Chile.pandradem@udec.cl

2 Universidad Alberto Hurtado, Santiago, Chile. macastrr@uahurtado.cl

Universidad de Chile.

4 Museo Chileno de Arte Precolombino, Santiago, Chile. caldunate@museoprecolombino.cl
} 
Formativo (Andrade et al. 2014; Constanzo 2013; Flores 2013; Loyola y Rebeco 2011; Urrea 2013).

Este artículo se enfoca en el estudio de cuatro individuos recuperados del sitio Copaca 1, ubicado a $30 \mathrm{~km}$ al sur de Tocopilla (Figura 1). Se trata de uno de los pocos sitios de la costa arreica que no ha sido huaqueado. Aquí se encontró una estructura subcircular de piedra (Figura 2) donde se recuperaron los individuos prácticamente completos. La ocupación del sitio ha sido fechada entre 7.000-4.500 a.p. (Castro et al. 2012).

A continuación se presenta una primera aproximación a los modos de vida de estos individuos que habitaron la costa arreica durante el período Arcaico IV (Llagostera 2005). Previniendo que se trata de una muestra pequeña, la información recuperada no necesariamente se puede aplicar para todos los grupos humanos que habitaron esta costa de más de $800 \mathrm{~km}$ de longitud.

\section{Antecedentes de los Contextos Funerarios}

El sitio Copaca $1\left(22^{\circ} 20^{\prime} \mathrm{S} ; 70^{\circ} 14^{\prime} \mathrm{W}\right)$ se encuentra emplazado a cielo abierto a $22 \mathrm{msm}$ adyacente al borde litoral actual a una distancia de $100 \mathrm{~m}$ del mar. Corresponde a un extenso conchal de $5.000 \mathrm{~m}^{2}$ con una densidad estratigráfica de 2,5 $\mathrm{m}$ de profundidad, y comprende una secuencia de al menos ocho eventos ocupacionales del Holoceno Medio, que habitaron el sitio como un asentamiento multifuncional. Los cuatro individuos
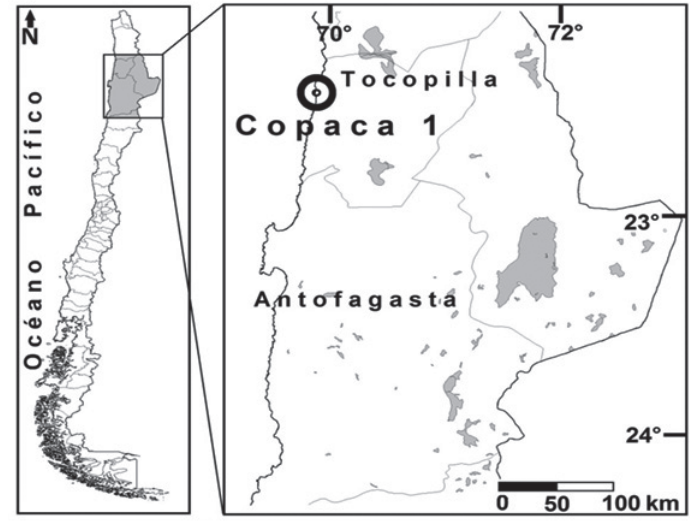

Figura 1. Ubicación geográfica del sitio arqueológico Copaca 1. Geographic location of the archaeological site Copaca 1.

recuperados se encontraron depositados en las capas 5 y 6 del conchal, fechadas por radiocarbono 14 en $5.240 \pm 25$ cal. a.p. (UGAM-8347) y $5.710 \pm 25$ cal a.p. (UGAM-8348), respectivamente (Castro et al. 2014; Peña-Villalobos et al. 2013). Además se dataron por el mismo método muestras óseas de cada uno de los esqueletos, existiendo coherencia cronológica tanto entre ellos como con las capas anteriormente mencionadas (Tabla 1).

Los cuatro individuos fueron encontrados al interior de la estructura mencionada. Dos de ellos (Individuos 1 y 4), encontrados en la capa 5, fueron dispuestos sobre un piso sello, a diferencia de lo que se ha planteado para el sitio Caleta Huelén

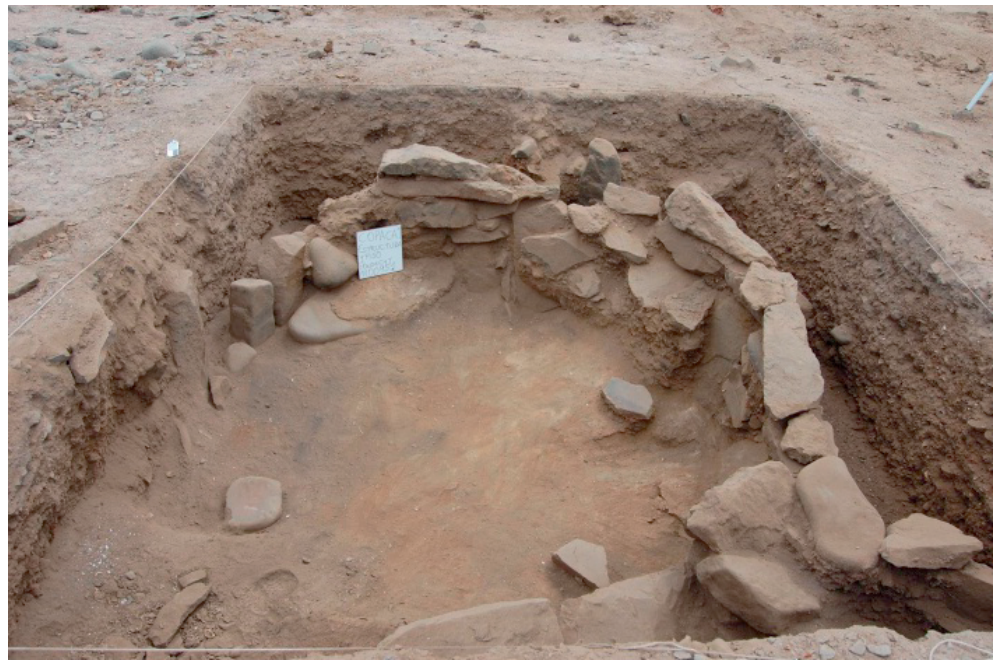

Figura 2. Estructura subcircular de piedra descubierta en el sitio arqueológico Copaca 1. Subcircular stone structure discovered in the archaeological site Copaca 1. 
Tabla 1. Resultados de University of Georgia Center for Applied Studies, 2013. Radiocarbon Human Osteological dates.

\begin{tabular}{cllccc}
\hline UGAM S\# & Identificación Muestra & Material & $\delta 13$ C, \%o & $\begin{array}{c}\text { Edad 14C } \\
\text { años a.p. }\end{array}$ & \pm \\
\hline 15623 & Copaca 1, Individuo 1 & Colágeno & $-12,0$ & 5.200 & 25 \\
15624 & Copaca 1, Individuo 2 & Colágeno & $-11,5$ & 5.220 & 25 \\
15625 & Copaca 1, Individuo 3 & Colágeno & $-11,9$ & 5.140 & 25 \\
15626 & Copaca 1, Individuo 4 & Colágeno & $-12,2$ & 5.150 & 25 \\
\hline
\end{tabular}

42 (Núñez et al. 1974), mientras que los otros dos (Individuos 2 y 3 ) se encontraban bajo este piso sello.

El individuo 1 se encontró depositado en posición decúbito dorsal, con las extremidades inferiores separadas y correctamente articulado en casi su totalidad. Estaba disturbado en el área de las costillas, la cintura escapular y vértebras cervicales, las que fueron desplazadas en forma articulada. En el espacio dejado por el desplazamiento de estas vértebras se colocaron dos conchas de erizos (Figuras 3 y 4). De acuerdo con el registro in situ y a la estratigrafía inalterada, se sugiere que este individuo no fue disturbado por agentes que actuaron después del abandono del sitio, sino que estas modificaciones fueron llevadas a cabo al momento del entierro, probablemente como parte de ritualidades funerarias novedosas en la costa arreica. Este contexto podría corresponder a una fase ceremonial del entierro. Considerando que no existen huellas de corte presentes en las vértebras y la forma en que se encontraban articuladas desde C3 a C7, no se puede establecer que se tratase de un ritual funerario asociado al descarnamiento, ni que el peso de los erizos pudo haber desplazado

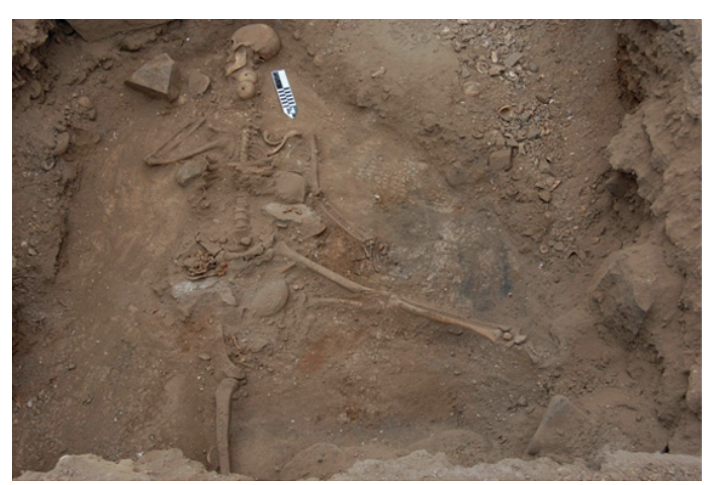

Figura 3. Vista general del contexto funerario del Individuo 1. General view of the funerary context of Individual 1. estas piezas óseas. Frente a esta situación debemos ser cautos, ya que la ausencia de evidencia similar en la zona de estudio no nos permite referirnos de manera más asertiva a la ritualidad expresada por estos grupos humanos.

El individuo 4 se encontró desarticulado y depositado entre las piernas del individuo 1 . Estas evidencias conducen a la hipótesis de que correspondería a un entierro secundario. Finalmente los individuos 2 y 3 se registraron en la capa 6 y corresponden a entierros secundarios, desarticulados

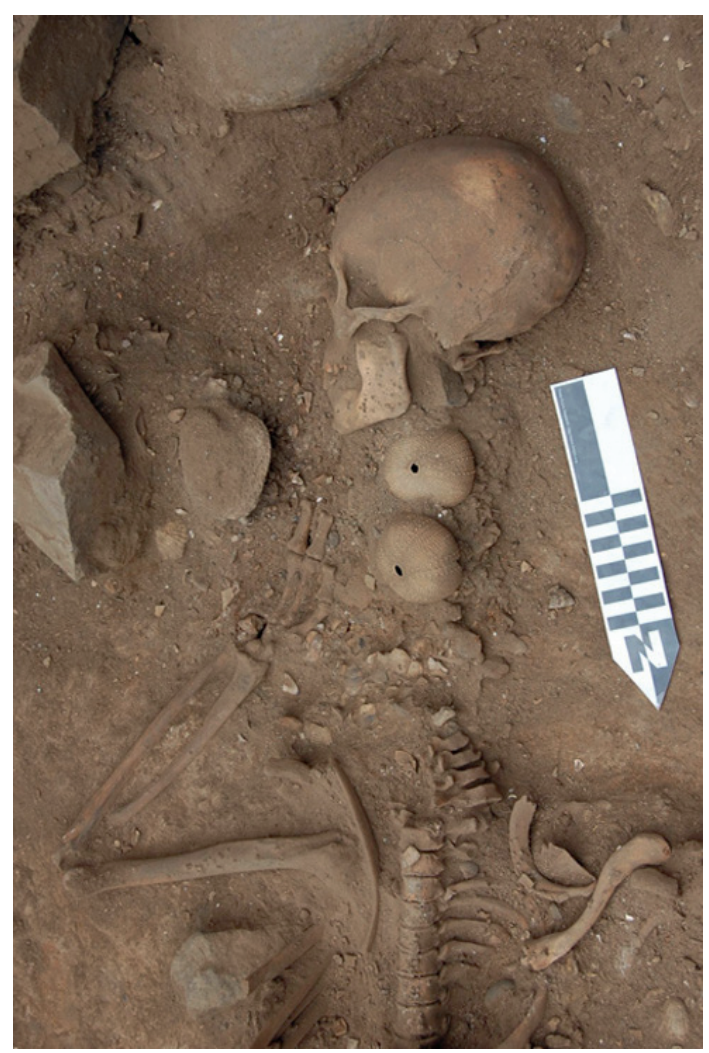

Figura 4. Detalle de las conchas de erizos ubicadas en la región cervical del Individuo 1.

Details of sea urchin shells located in the cervical region of Individual 1. 
y depositados en forma de "paquetes". A partir del inventario óseo (Tablas 2 y 3), podemos afirmar que los Individuos 1 y 2 presentan sobre el $75 \%$ de su registro óseo, mientras que el Individuo 3 se encontró medianamente completo y el Individuo 4 presentaba un registro óseo menor al 50\%.

\section{Metodología Bioarqueológica}

En primer término se realizaron inventarios de las unidades óseas y piezas dentales presentes en cada uno de los individuos (Tabla 3). Se ocuparon las recomendaciones propuestas por Bass (1987), sobre todo en el caso de los individuos que pertenecían a los contextos secundarios. Para la estimación de la edad se consideraron las categorías generales de edad propuestas por Buikstra y Ubelaker (1994), llevadas a cabo mediante la fusión de las epífisis de los huesos largos y de las vértebras sacras (Bass 1987; Buikstra y Ubelaker 1994; Campillo y Subirá 2004), la morfología esternal de la cuarta costilla (Iscan y Loth 1986), la morfología de la aurícula de los coxales (Lovejoy et al. 1985) y de la sínfisis púbica (Meindl et al. 1985), y el cierre de las suturas craneales (Hershkovitz et al. 1997).

La determinación de sexo fue realizada considerando los rasgos morfológicos observados en el cráneo y coxales (Bass 1987; Buikstra y Ubelaker 1994; Campillo y Subirá 2004). La determinación de la estatura se realizó de acuerdo con la medición de segmentos óseos descrita por Fully y Pineau (1960, en Krenzer 2006) y mediante la metodología descrita

Tabla 2. Inventario de unidades óseas I, según porcentaje de presencia (0: ausente, 1 : <25\%; 2: 25\%-50\%; 3: 50\%-75\% 4: < 75\%)

Osteological record, according its percentual presence (0: absent, 1: <25\%; 2: 25\%-50\%; 3: 50\%-75\% 4: <75\%).

\begin{tabular}{|c|c|c|c|c|}
\hline & $\begin{array}{c}\text { Individuo } \\
1\end{array}$ & $\begin{array}{c}\text { Individuo } \\
2\end{array}$ & $\begin{array}{c}\text { Individuo } \\
3\end{array}$ & $\begin{array}{c}\text { Individuo } \\
4\end{array}$ \\
\hline Cráneo & 4 & 4 & 3 & 3 \\
\hline Mandíbula & 4 & 4 & 3 & 0 \\
\hline Hioides & 0 & 0 & 0 & 0 \\
\hline Esternón & 1 & 4 & 3 & 0 \\
\hline Escápula derecha & 3 & 3 & 2 & 0 \\
\hline Escápula izquierda & 3 & 3 & 3 & 0 \\
\hline Clavícula derecha & 4 & 4 & 3 & 0 \\
\hline Clavícula izquierda & 4 & 2 & 2 & 0 \\
\hline Húmero derecho & 4 & 4 & 4 & 0 \\
\hline Húmero izquierdo & 4 & 4 & 3 & 3 \\
\hline Ulna derecha & 4 & 3 & 3 & 0 \\
\hline Ulna izquierda & 4 & 3 & 0 & 4 \\
\hline Radio derecho & 4 & 3 & 3 & 0 \\
\hline Radio izquierdo & 4 & 4 & 0 & 4 \\
\hline Mano derecha & 4 & 2 & 3 & 0 \\
\hline Mano izquierda & 4 & 2 & 0 & 2 \\
\hline Coxal derecho & 4 & 3 & 3 & 0 \\
\hline Coxal izquierdo & 4 & 3 & 3 & 0 \\
\hline Sacro & 4 & 4 & 4 & 1 \\
\hline Cóccix & 4 & 0 & 2 & 0 \\
\hline Fémur derecho & 4 & 4 & 4 & 4 \\
\hline Fémur izquierdo & 3 & 4 & 4 & 0 \\
\hline Patella derecha & 4 & 0 & 0 & 0 \\
\hline Patella izquierda & 0 & 0 & 4 & 0 \\
\hline Tibia derecha & 4 & 4 & 4 & 0 \\
\hline Tibia izquierda & 4 & 4 & 4 & 0 \\
\hline Fíbula derecha & 4 & 4 & 3 & 0 \\
\hline Fíbula izquierda & 4 & 4 & 3 & 0 \\
\hline Pie derecho & 3 & 2 & 3 & 0 \\
\hline Pie izquierdo & 3 & 2 & 3 & 0 \\
\hline
\end{tabular}


Tabla 3. Inventario de unidades óseas II, según porcentaje de presencia (0: ausente, $1:<25 \% ; 2: 25 \%-50 \%$; 3: $50 \%-75 \%$ : $<75 \%)$.

Osteological record according its porcentual presence (0: absent, 1 : $<25 \% ; 2: 25 \%-50 \%$; 3: $50 \%-75 \% 4:<75 \%)$.

\begin{tabular}{|c|c|c|c|c|}
\hline & $\begin{array}{c}\text { Individuo } \\
1\end{array}$ & $\begin{array}{c}\text { Individuo } \\
2\end{array}$ & $\begin{array}{c}\text { Individuo } \\
3\end{array}$ & $\begin{array}{c}\text { Individuo } \\
4\end{array}$ \\
\hline VC1 & 4 & 4 & 0 & 3 \\
\hline VC2 & 4 & 4 & 0 & 3 \\
\hline VC3 & 4 & 4 & 0 & 3 \\
\hline VC4 & 4 & 4 & 0 & 3 \\
\hline VC5 & 4 & 4 & 1 & 3 \\
\hline VC6 & 4 & 2 & 0 & 3 \\
\hline VC7 & 4 & 4 & 0 & 3 \\
\hline VT1 & 4 & 4 & 4 & 0 \\
\hline VT2 & 4 & 3 & 4 & 3 \\
\hline VT3 & 4 & 3 & 3 & 3 \\
\hline VT4 & 4 & 3 & 3 & 3 \\
\hline VT5 & 4 & 4 & 4 & 0 \\
\hline VT6 & 4 & 3 & 3 & 0 \\
\hline VT7 & 4 & 3 & 0 & 3 \\
\hline VT8 & 4 & 3 & 4 & 0 \\
\hline VT9 & 4 & 3 & 4 & 0 \\
\hline VT10 & 4 & 2 & 0 & 3 \\
\hline VT11 & 4 & 3 & 3 & 0 \\
\hline VT12 & 4 & 4 & 4 & 0 \\
\hline VL1 & 4 & 4 & 0 & 3 \\
\hline VL2 & 4 & 3 & 0 & 3 \\
\hline VL3 & 4 & 3 & 0 & 3 \\
\hline VL4 & 4 & 4 & 0 & 3 \\
\hline VL5 & 4 & 3 & 0 & 3 \\
\hline CD1 & 4 & 4 & 4 & 0 \\
\hline CD2 & 4 & 3 & 0 & 0 \\
\hline CD3 & 4 & 3 & 3 & 0 \\
\hline CD4 & 3 & 3 & 3 & 0 \\
\hline CD5 & 4 & 3 & 2 & 0 \\
\hline CD6 & 4 & 3 & 3 & 0 \\
\hline CD7 & 4 & 3 & 2 & 2 \\
\hline CD8 & 4 & 3 & 2 & 0 \\
\hline CD9 & 4 & 3 & 2 & 0 \\
\hline CD10 & 4 & 3 & 2 & 0 \\
\hline CD11 & 4 & 4 & 0 & 0 \\
\hline CD12 & 4 & 4 & 0 & 0 \\
\hline CI1 & 0 & 4 & 4 & 0 \\
\hline $\mathrm{CI} 2$ & 4 & 3 & 3 & 0 \\
\hline CI3 & 4 & 3 & 0 & 0 \\
\hline CI4 & 4 & 3 & 2 & 4 \\
\hline CI5 & 4 & 3 & 2 & 0 \\
\hline CI6 & 4 & 3 & 3 & 0 \\
\hline CI7 & 4 & 3 & 3 & 0 \\
\hline CI8 & 4 & 4 & 2 & 0 \\
\hline CI9 & 4 & 4 & 3 & 0 \\
\hline CI10 & 4 & 3 & 0 & 0 \\
\hline CI11 & 4 & 4 & 3 & 0 \\
\hline CI12 & 4 & 3 & 0 & 4 \\
\hline
\end{tabular}

por Genovés (1967, en Krenzer 2006) utilizando tibia y fémur.

El reconocimiento de las distintas condiciones patológicas y el grado de intensidad presente en los esqueletos consideró todos aquellos eventos traumáticos y enfermedades degenerativas, infecciosas y nutricionales, siguiendo las indicaciones de Mann y Hunt (2005), Aufderheide y MartínRodríguez (1998) y Buikstra y Ubelaker (1994). Adicionalmente, la identificación de signos de estrés ocupacional se realizó a partir de la observación de inserciones musculares con el fin de interpretar movimientos que pudieran haber sido realizados por los individuos (Bridges 1994; Constantinescu y Aspillaga 1991; Kennedy 1989; Ortner 2003).

Para el registro y análisis de las piezas dentales (Anexo 1) se consideró la presencia de caries, pérdida ante mortem de dientes, abscesos, cálculo dental e hipoplasia del esmalte, según las categorías de Buikstra y Ubelaker (1994), Chimenos (2004), Brothwell (1981) y Lukacs (1989). En el caso de los abscesos dentales se tuvo especial cuidado en su diagnóstico, tomando en cuenta las recomendaciones de Hillson (2008) y de Días y Tayles (1997).

Las muestras para el análisis de isótopos estables fueron extraídas de cortes de fémur de los individuos y enviadas al Center for Applied Isotope Studies, de la Universidad de Georgia, Estados Unidos y al Departamento de Antropología de la Universidad Estatal de California, Chico (Kline et al. 2013).

\section{Resultados}

Individuo 1: Presenta un buen estado de conservación. Corresponde a un individuo adulto masculino (35 y 45 años), con una estatura de $160 \pm 1,7 \mathrm{~cm}$. Las patologías observadas incluyen osteoartritis, enfermedades metabólicas, ocupacionales y afecciones dentales. Tiene marcadas inserciones musculares tanto en los miembros superiores como inferiores.

La osteoartritis afecta principalmente a los miembros superiores (Tablas 4 y 5) y columna vertebral, en grado moderado. Además, en las vértebras torácicas bajas y lumbares se observó la presencia de nódulos de Schmorl, lipping y osteofitos.

Se registraron patologías metabólicas, como criba orbitaria. Las enfermedades dentales (Tablas 6 y 7) muestran una ausencia total de caries e hipoplasia del esmalte, una leve concentración de cálculo dental, enfermedad periodontal y presencia de tres abscesos. El desgaste dental se muestra 
plano y severo, destruyendo el esmalte y dejando expuesta la dentina y algunas veces la cámara pulpar (Figura 5). Finalmente, la presencia de enfermedades asociadas a actividades productivas corresponde a exostosis auditiva externa incipiente en ambos meatos, facetas de acuclillamiento en las epífisis distales de ambas tibias y la presencia de una marcada retroversión en la epífisis proximal de ambos húmeros.

Individuo 2: Presenta en buen estado de conservación. Corresponde a un individuo de sexo masculino, 25 a 30 años, con una estatura de $165 \mathrm{~cm}$. En cuanto a condiciones patológicas son similares a las observadas en el individuo 1, como también el hecho de presentar marcadas inserciones musculares. Las enfermedades degenerativas articulares (Tablas 4 y 5 ) se concentran en columna vertebral y en menor medida en las extremidades; la articulación temporomandibular, expresada en forma de osteoartritis moderada. Además, gran parte de las vértebras torácicas y lumbares presentan lipping y osteofitos. En cuanto a la presencia de nódulos de Schmorl, solo se pudo constatar un caso en la novena vértebra torácica.

Las enfermedades metabólicas corresponden a criba orbitaria en ambas órbitas e hiperostosis porótica incipiente en ambos parietales. Las patologías dentales (Tablas 6 y 7) indican la presencia de dos abscesos (Figura 6) y leve presencia de cálculo dental, concentrado principalmente en las piezas dentales posteriores; hay ausencia total de caries y de hipoplasia del esmalte. El desgaste dental se muestra plano y severo, destruyendo el esmalte y dejando expuesta la dentina y a veces la cámara pulpar.

Las patologías asociadas a labores productivas corresponden a exostosis auditiva externa bilateral, facetas de acuclillamiento en ambas tibias y la presencia de una marcada retroversión en la epífisis proximal de ambos húmeros. También se observó en ambos fémures una hipervascularización en las epífisis distales.

Tabla 4. Registro de las patologías en piezas dentales superiores de los individuos adultos de Copaca 1. (3M: Tercer molar, 2M Segundo molar, 1M: Primer molar, 2PM: Segundo premolar, 1PM: primer premolar,

C: Canino, IC: Incisivo central, IL: Incisivo lateral) (ver Anexo 1).

Patological record of superior teeth of the adult indivuals from Copaca 1.

(3M: Thrid molar, 2M: Second molar, 1M: First molar, 2PM: Second premolar; 1PM: firts premolar, C: Canine, IL: Lateral incisor, IC: Central incisor). See Anex 1.

\begin{tabular}{|c|c|c|c|c|c|c|c|c|c|c|c|c|c|c|c|c|c|}
\hline \multirow{2}{*}{ Individuo } & & \multicolumn{8}{|c|}{ Piezas dentales derechas } & \multicolumn{8}{|c|}{ Piezas dentales izquierdas } \\
\hline & & $3 \mathrm{M}$ & $2 \mathrm{M}$ & $1 \mathrm{M}$ & $2 \mathrm{PM}$ & $1 \mathrm{PM}$ & $\mathrm{C}$ & IL & IC & IC & IL & $\mathrm{C}$ & $1 \mathrm{PM}$ & $2 \mathrm{PM}$ & $1 \mathrm{M}$ & $2 \mathrm{M}$ & $3 \mathrm{M}$ \\
\hline \multirow{8}{*}{1} & Estado alveolar & 1 & 1 & 1 & 1 & 1 & 1 & 1 & 1 & 1 & 0 & 0 & 0 & 1 & 1 & 2 & 2 \\
\hline & Estado dentario & 1 & 1 & 1 & 1 & 1 & 1 & 1 & 2 & 2 & 2 & 2 & 2 & 1 & 2 & 3 & 3 \\
\hline & Caries + grado & 1 & 1 & 1 & 1 & 1 & 1 & 1 & 1 & 1 & 1 & 1 & 1 & 1 & 1 & 0 & 0 \\
\hline & Reabsorción alveolar & 2 & 2 & 0 & 0 & 2 & 2 & 2 & 0 & 2 & 0 & 0 & 0 & 1 & 0 & 0 & 0 \\
\hline & Cálculo & 1 & 1 & 0 & 1 & 4 & 4 & 1 & 4 & 2 & 4 & 4 & 2 & 4 & 2 & 0 & 0 \\
\hline & Abscesos & 2 & 1 & 2 & 1 & 1 & 1 & 1 & 0 & 1 & 0 & 0 & 0 & 1 & 0 & 0 & 0 \\
\hline & Hipoplasia del esmalte & 1 & 1 & 1 & 1 & 1 & 1 & 1 & 1 & 1 & 1 & 1 & 1 & 1 & 0 & 0 & 0 \\
\hline & Desgaste & 2 & 4 & $5+$ & 5 & 5 & 5 & 5 & 5 & 4 & 4 & 5 & $5++$ & 5 & $5++$ & 0 & 0 \\
\hline \multirow{8}{*}{2} & Estado alveolar & 1 & 1 & 1 & 1 & 1 & 1 & 1 & 1 & 1 & 1 & 1 & 1 & 1 & 1 & 1 & 1 \\
\hline & Estado dentario & 1 & 1 & 1 & 1 & 1 & 1 & 1 & 1 & 1 & 2 & 1 & 1 & 1 & 1 & 1 & 1 \\
\hline & Caries + grado & 1 & 1 & 1 & 1 & 1 & 1 & 1 & 1 & 1 & 4 & 1 & 1 & 1 & 1 & 1 & 1 \\
\hline & Reabsorción alveolar & 1 & 1 & 2 & 2 & 4 & 4 & 2 & 2 & 4 & 4 & 2 & 2 & 1 & 1 & 1 & 1 \\
\hline & Cálculo & 1 & 1 & 1 & 1 & 1 & 1 & 1 & 1 & 1 & 4 & 2 & 1 & 1 & 1 & 1 & 1 \\
\hline & Abscesos & 1 & 1 & 1 & 1 & 1 & 1 & 1 & 1 & 1 & 5 & 1 & 1 & 1 & 3 & 4 & 2 \\
\hline & Hipoplasia del esmalte & 1 & 1 & 1 & 1 & 1 & 1 & 1 & 1 & 1 & 1 & 1 & 1 & 1 & 1 & 1 & 1 \\
\hline & Desgaste & 1 & 3 & 6 & 5 & 4 & 3 & 6 & 6 & 6 & -- & 6 & 5 & 4 & 5 & 4 & 2 \\
\hline \multirow{8}{*}{3} & Estado alveolar & 1 & 1 & 1 & 1 & 1 & 1 & 1 & 1 & 1 & 1 & 1 & 1 & 1 & 1 & 1 & 1 \\
\hline & Estado dentario & 1 & 1 & 1 & 1 & 1 & 1 & 1 & 1 & 1 & 1 & 1 & 1 & 1 & 1 & 1 & 1 \\
\hline & Caries + grado & 1 & 1 & 1 & 1 & 1 & 1 & 1 & 1 & 1 & 1 & 1 & 1 & 1 & 1 & 1 & 1 \\
\hline & Reabsorción alveolar & 0 & 1 & 1 & 1 & 1 & 1 & 1 & 1 & 0 & 1 & 1 & 1 & 1 & 1 & 1 & 1 \\
\hline & Cálculo & 1 & 1 & 1 & 1 & 1 & 1 & 1 & 1 & 1 & 1 & 1 & 1 & 1 & 1 & 1 & 1 \\
\hline & Abscesos & 2 & 2 & 3 & 2 & 2 & 2 & 2 & 1 & 2 & 2 & 3 & 2 & 2 & 4 & 2 & 3 \\
\hline & Hipoplasia del esmalte & 1 & 1 & 1 & 1 & 1 & 1 & 1 & 1 & 1 & 1 & 1 & 1 & 1 & 1 & 1 & 1 \\
\hline & Desgaste & 2 & 2 & 4 & 3 & 3 & 3 & 2 & 2 & 4 & 3 & 3 & 3 & 3 & 4 & 3 & 2 \\
\hline
\end{tabular}


Tabla 5. Registro de las patologías en piezas dentales inferiores de los individuos adultos de Copaca 1.

(3M: Tercer molar, 2M Segundo molar, 1M: Primer molar, 2PM: Segundo premolar, 1PM: primer premolar,

C: Canino, IC: Incisivo central, IL: Incisivo lateral). Ver Anexo 1.

Patological record of inferior teeth of the adult individuals from Copaca 1.

(3M: Third molar, 2M: Second molar, 1M: First molar, 2PM: Second premolar; 1PM: first premolar,

C: Canine, IL: Lateral incisor, IC: Central incisor). See Anex 1.

\begin{tabular}{|c|c|c|c|c|c|c|c|c|c|c|c|c|c|c|c|c|c|}
\hline \multirow{2}{*}{ Individuo } & & \multicolumn{8}{|c|}{ Piezas dentales derechas } & \multicolumn{8}{|c|}{ Piezas dentales izquierdas } \\
\hline & & $3 \mathrm{M}$ & $2 \mathrm{M}$ & $1 \mathrm{M}$ & $2 \mathrm{PM}$ & $1 \mathrm{PM}$ & $\mathrm{C}$ & IL & IC & $\mathrm{IC}$ & IL & $\mathrm{C}$ & 1PM & $2 \mathrm{PM}$ & $1 \mathrm{M}$ & $2 \mathrm{M}$ & $3 \mathrm{M}$ \\
\hline \multirow{8}{*}{1} & Estado alveolar & 1 & 1 & 1 & 1 & 1 & 1 & 3 & 3 & 3 & 1 & 1 & 1 & 1 & 1 & 1 & 1 \\
\hline & Estado dentario & 1 & 1 & 1 & 1 & 1 & 1 & 2 & 4 & 2 & 1 & 1 & 1 & 1 & 1 & 1 & 1 \\
\hline & Caries + grado & 1 & 1 & 1 & 1 & 1 & 1 & 1 & 0 & 1 & 1 & 1 & 1 & 1 & 1 & 1 & 1 \\
\hline & Reabsorción alveolar & 1 & 2 & 2 & 0 & 1 & 2 & 0 & 0 & 0 & 1 & 2 & 0 & 1 & 2 & 2 & 2 \\
\hline & Cálculo & 3 & 3 & 3 & 1 & 1 & 2 & 1 & 0 & 1 & 2 & 2 & 1 & 1 & 1 & 2 & 1 \\
\hline & Abscesos & 1 & 1 & 1 & 1 & 1 & 1 & 0 & 0 & 0 & 2 & 1 & 1 & 1 & 1 & 1 & 1 \\
\hline & Hipoplasia del esmalte & 1 & 1 & 1 & 1 & 1 & 1 & 1 & 0 & 1 & 1 & 1 & 1 & 1 & 1 & 1 & 1 \\
\hline & Desgaste & 4 & $5+$ & 5 & 6 & 6 & 5 & 5 & 0 & 5 & 5 & 5 & $5++$ & $5++$ & $5++$ & 5 & 2 \\
\hline \multirow{8}{*}{2} & Estado alveolar & 1 & 1 & 1 & 1 & 1 & 1 & 1 & 1 & 1 & 1 & 1 & 1 & 1 & 1 & 2 & 1 \\
\hline & Estado dentario & 1 & 1 & 1 & 1 & 1 & 1 & 2 & 2 & 2 & 1 & 1 & 1 & 1 & 1 & 3 & 1 \\
\hline & Caries + grado & 1 & 1 & 1 & 1 & 1 & 1 & 4 & 4 & 4 & 1 & 1 & 1 & 1 & 1 & 4 & 1 \\
\hline & Reabsorción alveolar & 1 & 1 & 2 & 1 & 2 & 2 & 0 & 0 & 0 & 2 & 2 & 2 & 1 & 1 & 0 & 1 \\
\hline & Cálculo & 1 & 1 & 1 & 1 & 1 & 1 & 4 & 4 & 4 & 1 & 1 & 1 & 1 & 1 & 4 & 1 \\
\hline & Abscesos & 1 & 1 & 1 & 1 & 1 & 1 & 5 & 5 & 5 & 1 & 1 & 1 & 1 & 2 & 5 & 3 \\
\hline & Hipoplasia del esmalte & 1 & 1 & 1 & 1 & 1 & 1 & 1 & 1 & 2 & 2 & 1 & 1 & 1 & 1 & 1 & 1 \\
\hline & Desgaste & 2 & 4 & 6 & 5 & 5 & 6 & -- & -- & -- & 6 & 7 & 6 & 5 & 5 & -- & 2 \\
\hline \multirow{8}{*}{3} & Estado alveolar & 4 & 1 & 1 & 1 & 1 & 1 & 1 & 1 & 1 & 1 & 1 & 1 & 1 & 4 & 1 & 1 \\
\hline & Estado dentario & 2 & 1 & 2 & 2 & 2 & 4 & 1 & 1 & 1 & 1 & 1 & 1 & 1 & 2 & 2 & 1 \\
\hline & Caries + grado & 1 & 1 & 1 & 1 & 1 & 1 & 1 & 1 & 1 & 1 & 1 & 1 & 1 & 1 & 1 & 1 \\
\hline & Reabsorción alveolar & 0 & 1 & 0 & 0 & 0 & 0 & 0 & 1 & 1 & 1 & 1 & 1 & 1 & 0 & 0 & 1 \\
\hline & Cálculo & 1 & 1 & 1 & 2 & 1 & 1 & 1 & 1 & 1 & 1 & 1 & 1 & 1 & 1 & 1 & 1 \\
\hline & Abscesos & 2 & 3 & 3 & 2 & 2 & 0 & 2 & 2 & 2 & 3 & 3 & 3 & 3 & 3 & 2 & 2 \\
\hline & Hipoplasia del esmalte & 0 & 1 & 0 & 0 & 0 & 0 & 1 & 1 & 1 & 1 & 1 & 1 & 1 & 0 & 0 & 1 \\
\hline & Desgaste & 1 & 3 & 2 & 2 & 3 & -- & 5 & 6 & 6 & 5 & 4 & 3 & 3 & 4 & 3 & 1 \\
\hline
\end{tabular}

Individuo 3: Se trata de un individuo de sexo femenino en buen estado de conservación (Tablas 4 y 5). Su edad oscilaría entre los 20 y 25 años de edad y su estatura alrededor de 152 $\mathrm{cm}$. Las patologías observadas presentan algunas diferencias respecto de los individuos anteriores. Además, exhibe moderadas inserciones en miembros superior e inferior.

Referente a las enfermedades degenerativas articulares, estas se expresan como una leve osteoartritis en las articulaciones de la cadera (Tabla 4), estando estas ausentes en el esqueleto axial (Tabla 5). Las enfermedades metabólicas corresponden a criba orbitaria en la órbita izquierda. Además se detectó una severa osteomielitis en la tibia derecha. Las enfermedades dentales, por su parte, muestran una ausencia de caries y abscesos (Tablas 6 y 7). Se observó un caso de hipoplasia del esmalte y la presencia de cálculo leve a moderado en todas las piezas dentales. En cuanto al desgaste dental, este se muestra plano y leve, y en la mayoría de los casos compromete solo el esmalte. Además se detectó la presencia de un alveolo de un incisivo central deciduo retenido que fue perdido post mortem. Finalmente, en lo correspondiente a las patologías asociadas a actividades productivas, se puede decir que este individuo presentaba facetas de acuclillamiento en ambas tibias.

Individuo 4: Corresponde a un individuo de sexo indeterminado en buen estado de preservación, siendo analizado previamente por Loyola y Rebeco (2011). Se determinó una edad de 3 a 5 años y la presencia de enfermedades se limita a criba femoral bilateral. Se pudo observar además la presencia de entesis en la línea áspera de ambos fémures. 


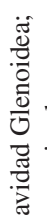

范

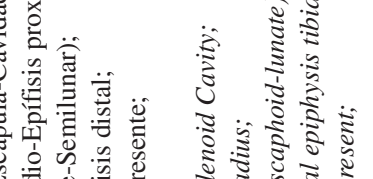

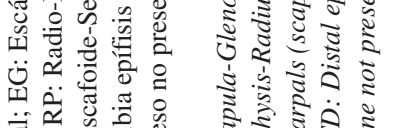

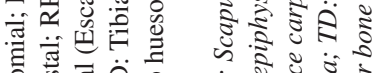

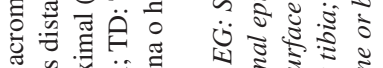

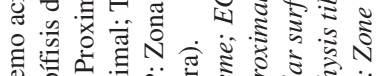

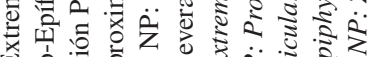

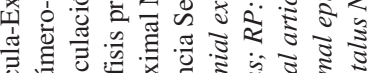

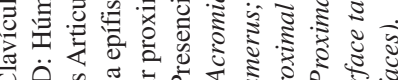

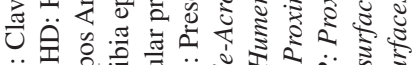
ii

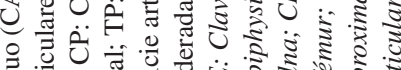

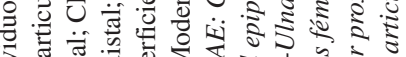

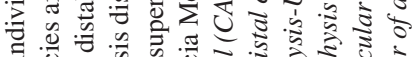

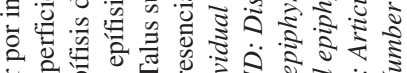

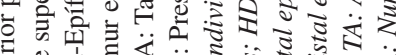

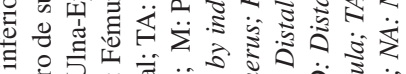

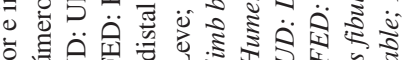

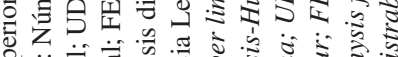

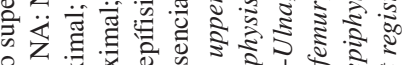

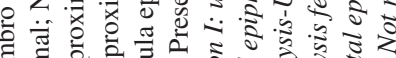

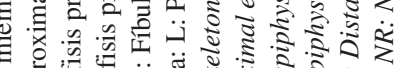

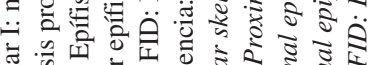

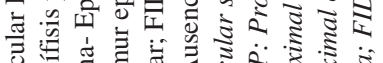

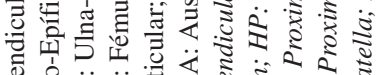

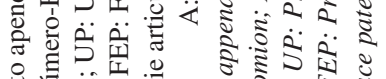

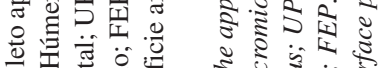

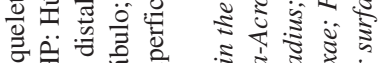

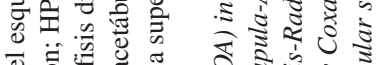
ब. 0े

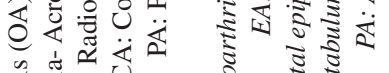

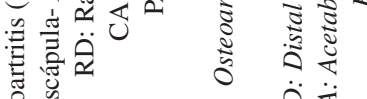

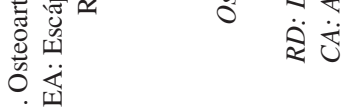

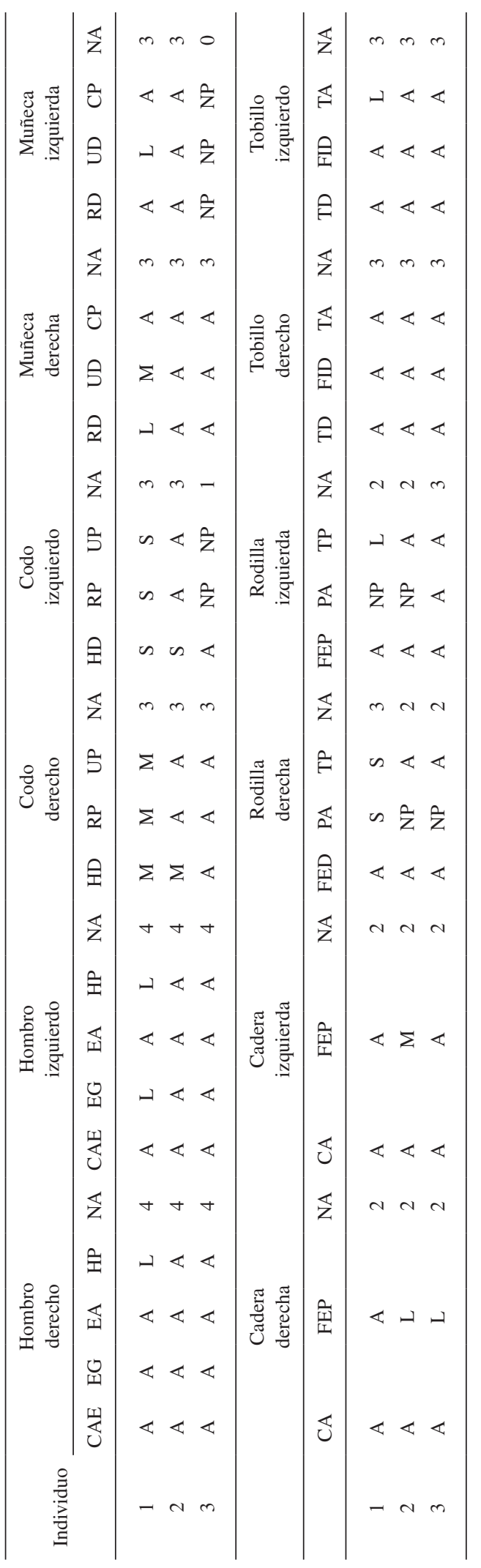


Tabla 7. Registro de las patologías en piezas dentales inferiores de los individuos adultos de Copaca 1.

(3M: Tercer molar, 2M Segundo molar, 1M: Primer molar, 2PM: Segundo premolar, 1PM: primer premolar,

C: Canino, IC: Incisivo central, IL: Incisivo lateral). Ver Anexo 1.

Patological record of inferior teeth of the adult individuals from Copaca 1.

(3M: Third molar, 2M: Second molar, 1M: First molar, 2PM: Second premolar; 1PM: first premolar,

C: Canine, IL: Lateral incisor, IC: Central incisor). See Anex 1.

\begin{tabular}{|c|c|c|c|c|c|c|c|c|c|c|c|c|c|c|c|c|c|}
\hline \multirow{2}{*}{ Individuo } & & \multicolumn{8}{|c|}{ Piezas dentales derechas } & \multicolumn{8}{|c|}{ Piezas dentales izquierdas } \\
\hline & & $3 \mathrm{M}$ & $2 \mathrm{M}$ & $1 \mathrm{M}$ & $2 \mathrm{PM}$ & $1 \mathrm{PM}$ & $\mathrm{C}$ & IL & IC & IC & IL & $\mathrm{C}$ & 1PM & $2 \mathrm{PM}$ & $1 \mathrm{M}$ & $2 \mathrm{M}$ & $3 \mathrm{M}$ \\
\hline \multirow{8}{*}{1} & Estado alveolar & 1 & 1 & 1 & 1 & 1 & 1 & 3 & 3 & 3 & 1 & 1 & 1 & 1 & 1 & 1 & 1 \\
\hline & Estado dentario & 1 & 1 & 1 & 1 & 1 & 1 & 2 & 4 & 2 & 1 & 1 & 1 & 1 & 1 & 1 & 1 \\
\hline & Caries + grado & 1 & 1 & 1 & 1 & 1 & 1 & 1 & 0 & 1 & 1 & 1 & 1 & 1 & 1 & 1 & 1 \\
\hline & Reabsorción alveolar & 1 & 2 & 2 & 0 & 1 & 2 & 0 & 0 & 0 & 1 & 2 & 0 & 1 & 2 & 2 & 2 \\
\hline & Cálculo & 3 & 3 & 3 & 1 & 1 & 2 & 1 & 0 & 1 & 2 & 2 & 1 & 1 & 1 & 2 & 1 \\
\hline & Abscesos & 1 & 1 & 1 & 1 & 1 & 1 & 0 & 0 & 0 & 2 & 1 & 1 & 1 & 1 & 1 & 1 \\
\hline & Hipoplasia del esmalte & 1 & 1 & 1 & 1 & 1 & 1 & 1 & 0 & 1 & 1 & 1 & 1 & 1 & 1 & 1 & 1 \\
\hline & Desgaste & 4 & $5+$ & 5 & 6 & 6 & 5 & 5 & 0 & 5 & 5 & 5 & $5++$ & $5++$ & $5++$ & 5 & 2 \\
\hline \multirow{8}{*}{2} & Estado alveolar & 1 & 1 & 1 & 1 & 1 & 1 & 1 & 1 & 1 & 1 & 1 & 1 & 1 & 1 & 2 & 1 \\
\hline & Estado dentario & 1 & 1 & 1 & 1 & 1 & 1 & 2 & 2 & 2 & 1 & 1 & 1 & 1 & 1 & 3 & 1 \\
\hline & Caries + grado & 1 & 1 & 1 & 1 & 1 & 1 & 4 & 4 & 4 & 1 & 1 & 1 & 1 & 1 & 4 & 1 \\
\hline & Reabsorción alveolar & 1 & 1 & 2 & 1 & 2 & 2 & 0 & 0 & 0 & 2 & 2 & 2 & 1 & 1 & 0 & 1 \\
\hline & Cálculo & 1 & 1 & 1 & 1 & 1 & 1 & 4 & 4 & 4 & 1 & 1 & 1 & 1 & 1 & 4 & 1 \\
\hline & Abscesos & 1 & 1 & 1 & 1 & 1 & 1 & 5 & 5 & 5 & 1 & 1 & 1 & 1 & 2 & 5 & 3 \\
\hline & Hipoplasia del esmalte & 1 & 1 & 1 & 1 & 1 & 1 & 1 & 1 & 2 & 2 & 1 & 1 & 1 & 1 & 1 & 1 \\
\hline & Desgaste & 2 & 4 & 6 & 5 & 5 & 6 & -- & -- & -- & 6 & 7 & 6 & 5 & 5 & -- & 2 \\
\hline \multirow{8}{*}{3} & Estado alveolar & 4 & 1 & 1 & 1 & 1 & 1 & 1 & 1 & 1 & 1 & 1 & 1 & 1 & 4 & 1 & 1 \\
\hline & Estado dentario & 2 & 1 & 2 & 2 & 2 & 4 & 1 & 1 & 1 & 1 & 1 & 1 & 1 & 2 & 2 & 1 \\
\hline & Caries + grado & 1 & 1 & 1 & 1 & 1 & 1 & 1 & 1 & 1 & 1 & 1 & 1 & 1 & 1 & 1 & 1 \\
\hline & Reabsorción alveolar & 0 & 1 & 0 & 0 & 0 & 0 & 0 & 1 & 1 & 1 & 1 & 1 & 1 & 0 & 0 & 1 \\
\hline & Cálculo & 1 & 1 & 1 & 2 & 1 & 1 & 1 & 1 & 1 & 1 & 1 & 1 & 1 & 1 & 1 & 1 \\
\hline & Abscesos & 2 & 3 & 3 & 2 & 2 & 0 & 2 & 2 & 2 & 3 & 3 & 3 & 3 & 3 & 2 & 2 \\
\hline & Hipoplasia del esmalte & 0 & 1 & 0 & 0 & 0 & 0 & 1 & 1 & 1 & 1 & 1 & 1 & 1 & 0 & 0 & 1 \\
\hline & Desgaste & 1 & 3 & 2 & 2 & 3 & -- & 5 & 6 & 6 & 5 & 4 & 3 & 3 & 4 & 3 & 1 \\
\hline
\end{tabular}

\section{Isótopos Estables}

Se enviaron muestras óseas (fémur) de los Individuos 1, 2 y 3 a CAIS, de la Universidad de Georgia y a la Universidad de California, Chico, Departamento de Antropología (Kline et al. 2013) (Tabla 8). Cabe hacer notar la alta correspondencia entre los resultados de ambos laboratorios. Ellos demostraron un altísimo índice de nitrógeno por el gran consumo de proteínas provenientes de la dieta marina. Lo mismo ocurre con los valores de carbono y los de espaciamiento entre apatita y colágeno, pues estos últimos varían entre 2,3 y 4,2, bastante inferiores a aquellos que exhiben dietas terrestres con consumo de plantas $\mathrm{C} 4$.

Por lo exiguo de la muestra, estimamos que sería arriesgado mostrar otros resultados conducentes a obtener, por ejemplo, diferencias de dieta por sexo o edad, entre otros.

\section{Discusión}

\section{Patologías}

Existen algunas enfermedades que parecen transversales a los individuos analizados y otras que parecen tener relación con el sexo de los mismos. Dentro del primer caso se pueden incluir las enfermedades metabólicas manifestadas en forma de criba orbitaria, hiperostosis porótica y criba femoral. Tradicionalmente se ha ligado esta patología a situaciones de estrés causadas por la privación de nutrientes y anemia, las que podrían tener su origen en factores dietarios y no dietarios, 


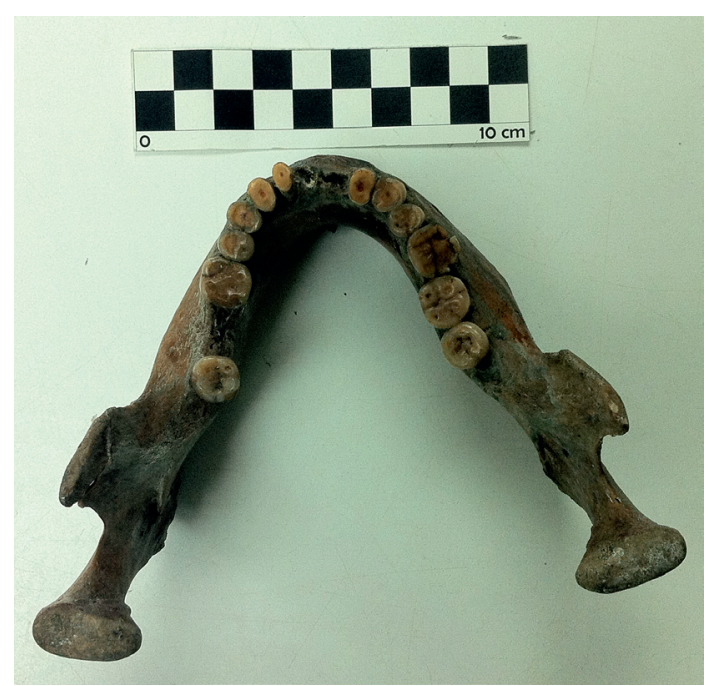

Figura 5. Desgaste de las piezas dentales inferiores del Individuo 1. Wear of the lower teeth of Individual 1.

como la falta de ingesta de comidas ricas en hierro, parásitos, diarreas infantiles (Larsen 1995:199; Rothschild 2000; Stuart-Macadam 1989, 2005) y por transmisión genética (Sisson y Lund 1958; Walker 1985), siendo tradicionalmente asociada a las poblaciones productoras de alimentos. Trabajos más recientes han indicado que la presencia de estas patologías no estarían ligadas a una anemia, sino a la falta de consumo de vitaminas C, B12 y B9 (Walker et al. 2009). En el caso de la muestra analizada, se puede argumentar que podrían aparecer estas condiciones patológicas debido a la falta de consumo de alimentos ricos en vitamina $\mathrm{C}$, aunque no aparecen huellas patológicas óseas ligadas a esta deficiencia (Ortner 2003). Por su parte, la vitamina B9 está presente en vegetales de hojas verdes, algunas frutas y legumbres (Allen 2008), sin consumo por parte de los individuos analizados aquí. Finalmente, la presencia de alto consumo de proteína marina, rica en vitamina $\mathrm{B} 12$, nos ocasiona un panorama de cierta dificultad interpretativa. Sin embargo, se ha podido determinar que en las poblaciones cazadoras recolectoras de zonas litorales marinas la presencia de estas patologías estaría relacionada con la ingesta del parásito Diphyllobothrium pacificum, que se encuentra en peces y en lobos marinos que son consumidos crudos (Reinhard y Urban 2003), lo que se compadece con la gran cantidad de fauna ictiológica recuperada en el sitio. Otra fuente de contagio de dicho parásito es por medio del consumo de carne de lobo marino, cuyos restos también forman parte del registro arqueológico del sitio y que, según referencias etnohistóricas, era utilizado como parte importante de la dieta e incluso para saciar la sed (Lizárraga 1968). Todo lo anterior nos hace pensar que los individuos recuperados de Copaca 1 habrían estado expuestos a una alta carga parasitaria producto de su dieta marina. Considerando todo lo anterior creemos que tanto la ingesta en la dieta de este parásito, que privaría a los individuos de la vitamina B12, como la ausencia de consumo de vitamina B9 sería la causa de estas enfermedades, como se ha planteado para otras poblaciones con modos de vida similares (Alfonso et al. 2011).

La presencia de enfermedades metabólicas aparece en todos los individuos analizados. Ello puede interpretarse en términos de un acceso a un mismo tipo de dieta por parte de la población, donde no existirían diferencias en cuanto a sexo ni edad. De la misma forma, se podría afirmar que la exposición a estos parásitos por parte de estos grupos costeros partiría a una edad temprana. Es importante mencionar que, para el caso de la muestra analizada para Caleta Huelén 42, también existen evidencias de este tipo de enfermedades (Cocilovo et al. 2005) aunque en bajas frecuencias.

En cuanto a las enfermedades degenerativas articulares estas se encuentran con una mayor expresión en los individuos adultos masculinos, en particular en los miembros superiores, y podrían relacionarse con actividades ligadas al uso de remos para la navegación (Jurmain y Kilgore 1995; Molnar 2006) y/o el uso de arpones u otros elementos arrojadizos para la caza (Kennedy 1989; Molnar 2006). Si bien la expresión de las osteoartropatías presenta limitaciones en su relación directa con las actividades (Henderson y Cardoso 2013), nuestra propuesta en cuanto a la navegación se sustenta en la evidencia tanto artefactual como faunística que se presenta en el sitio, la que nos habla de una explotación de recursos que se encuentran en una dimensión que supera el ámbito de la extracción más allá de la línea intermareal (Castro et al. 2015).

Por su parte, la expresión de estas patologías en la columna vertebral podrían estar ligadas a la carga de peso extracorporal sobre la espalda (Kennedy 1989; Larsen 1997:163-164), provocando un estrés mecánico constante (Redlund-Johnell y Larsson 1993; Sales et al. 2007). Se podría inferir una cierta similitud en las actividades realizadas por los individuos masculinos, presentando patologías en grados de expresión moderada y algunas veces 


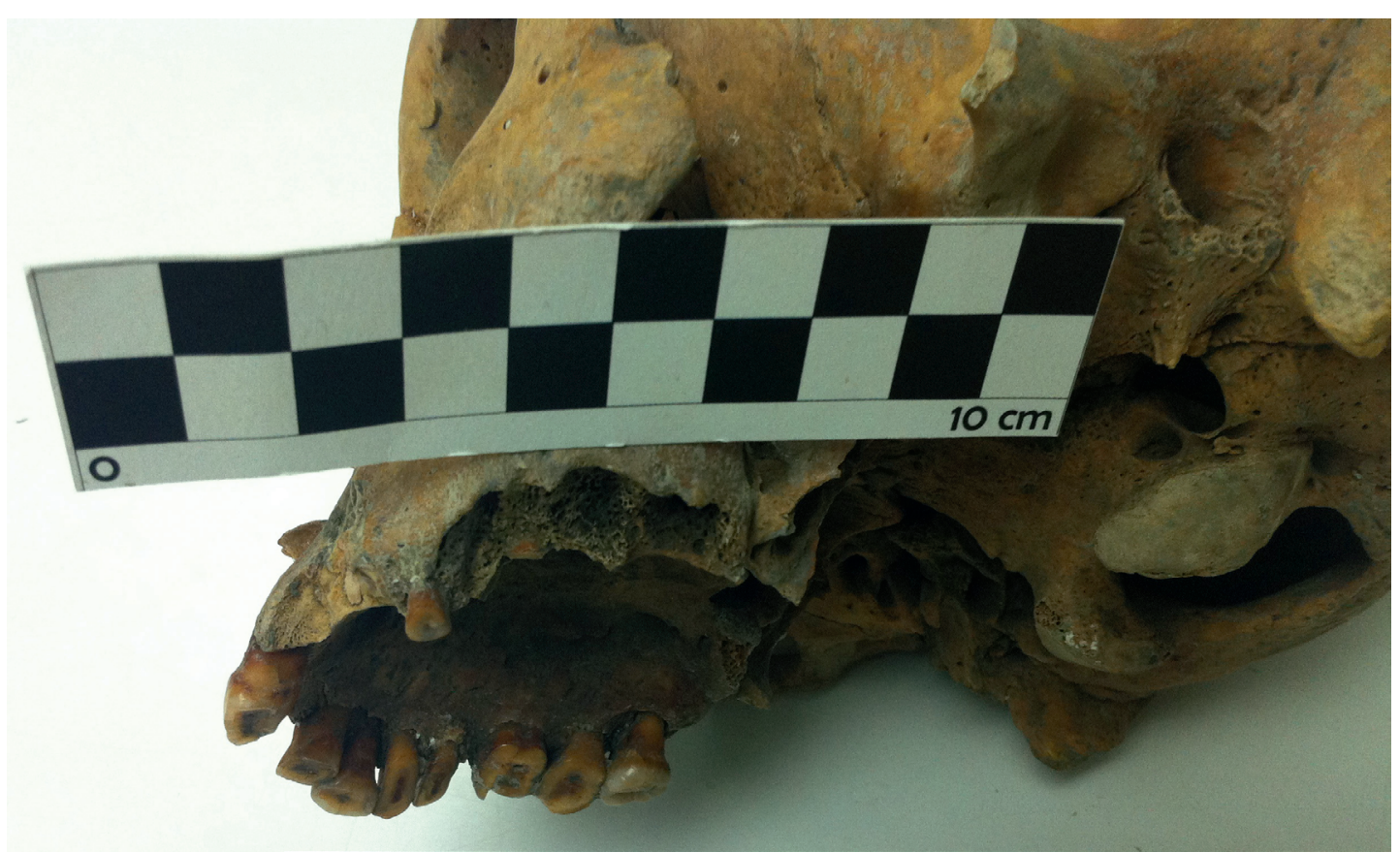

Figura 6. Abscesos observados en el maxilar del Individuo 2.

Abscesses observed in the maxilla of Individual 2.

Tabla 8. Líneas superiores: análisis de isótopos estables realizados por Kline et al. (2013).

Líneas inferiores: análisis de isótopos estables realizados en el CAIS, Universidad de Georgia.

Upper lines: stable isotope analysis performed by Kline et al. (2013).

Lower lines: stable isotope analysis performed on CAIS, University of Georgia.

\begin{tabular}{|c|c|c|c|c|c|c|c|c|}
\hline Muestra & $\begin{array}{l}\text { Cód. } \\
\text { Lab. }\end{array}$ & $\begin{array}{c}\delta 13 \\
\text { col \%o }\end{array}$ & $\begin{array}{c}\delta 15 \mathrm{~N} \\
\text { col. } \% o\end{array}$ & $\begin{array}{c}\delta 13 \mathrm{C} \\
\text { apat. } \\
\% o\end{array}$ & $\begin{array}{l}\text { Espaciamiento } \\
\text { apatita-colágeno }\end{array}$ & $\begin{array}{c}\delta 180 \\
\text { apat. } \\
\% \text { o }\end{array}$ & $\begin{array}{c}\text { Razón } \\
\text { C/N }\end{array}$ & $\begin{array}{c}\% \mathrm{Col} \\
\text { entregado }\end{array}$ \\
\hline \multirow{2}{*}{$\begin{array}{c}\text { Individuo } 1 \\
\text { masculino }\end{array}$} & $\begin{array}{c}\text { Kline et al. } \\
2013\end{array}$ & $-11,9$ & 24,4 & $-9,6$ & 2,3 & $-2,5$ & 3,3 & 5,4 \\
\hline & $\begin{array}{l}\text { UGAM } \\
13122\end{array}$ & $-12,3$ & 24,2 & $-10,2$ & & $-3,6$ & & \\
\hline \multirow{2}{*}{$\begin{array}{l}\text { Individuo } 2 \\
\text { masculino }\end{array}$} & $\begin{array}{c}\text { Kline et al. } \\
2013\end{array}$ & $-11,9$ & 23,7 & $-8,8$ & 3,1 & $-2,9$ & 3,3 & 3,2 \\
\hline & $\begin{array}{l}\text { UGAM } \\
13123\end{array}$ & $-11,8$ & 23,6 & $-9,7$ & & $-3,9$ & & \\
\hline \multirow{2}{*}{$\begin{array}{c}\text { Individuo } 3 \\
\text { femenino }\end{array}$} & $\begin{array}{c}\text { Kline et al. } \\
2013\end{array}$ & $-12,8$ & 23,5 & $-8,7$ & 4,2 & $-3,0$ & 3,3 & 3,6 \\
\hline & $\begin{array}{l}\text { UGAM } \\
13124\end{array}$ & $-12,3$ & 23,6 & $-9,5$ & & $-1,6$ & & \\
\hline
\end{tabular}


severa. Es posible que los hombres hayan realizado labores extractivas ligadas a la pesca y navegación, además de la carga de peso. En el caso del individuo femenino, este presenta lesiones leves o ausentes que también podrían explicarse por la juventud y/o sexo del individuo. Sin embargo, debido al tamaño de la muestra no podemos ser concluyentes respecto de si los individuos femeninos habrían estado exentos de estas actividades.

Las patologías dentales sugieren una dieta dura y/o rica en partículas abrasivas, observándose niveles importantes de desgaste dental, que dejó expuesta la dentina y en ocasiones la cámara pulpar. No hay evidencias de caries ni de hipoplasia del esmalte y existen bajos niveles de depositación de cálculo. Es probable que el marcado desgaste dental estaría repercutiendo en la ausencia de caries y en el registro efectivo de hipoplasia del esmalte. En consecuencia, se puede afirmar que la presencia de estas patologías es coincidente con aquellas descritas para grupos cazadores-recolectores marinos (Alfonso et al. 2008; Flores 2013; Quevedo 1998), lo que se ha confirmado por los estudios de isótopos estables, que indican la dependencia de proteínas marinas. La ausencia de caries es consecuencia de una baja ingesta de hidratos de carbono en la dieta.

Solo en el individuo femenino se presenta una enfermedad infecciosa en forma de osteomielitis, infección inespecífica, por lo que no se puede establecer cuál fue su origen (Larsen 1995, 1997). Considerando la muestra analizada, no podemos establecer la prevalencia de esta u otras enfermedades infecciosas para la muestra de Copaca 1; no obstante, la información para Caleta Huelén 42 indica que un poco más del $15 \%$ de los individuos analizados presentaba huellas de esta patología, estando presente tanto en hombres como en mujeres (Cocilovo et al. 2005).

\section{Actividades productivas}

Se constató la presencia de exostosis auditiva externa, que ha sido establecida para las poblaciones del extremo norte de Chile, como característica de los individuos masculinos que estuvieron expuestos a condiciones de agua fría como consecuencia de actividades de buceo para obtener mariscos (Kennedy 1989; Standen et al. 1995, 1997). De acuerdo con la muestra analizada, son justamente los individuos masculinos de Copaca 1 los que presentan esta patología; por tanto se puede asumir que eran ellos los encargados de realizar las actividades ligadas a la extracción de recursos en profundidades.

En cuanto a las inserciones musculares, todos los individuos analizados presentan huellas de haber incurrido en actividades con una alta demanda muscular, tanto en miembros superiores como inferiores. No obstante, algunas diferencias deben ser destacadas. En los individuos masculinos se observó la presencia de retroversión de las epífisis proximales de húmeros, lo que no fue observado en el individuo femenino (Figura 7). La presencia de estas inserciones musculares en los miembros superiores se ha establecido que correspondería a actividades ligadas con el uso de remos para la navegación (Kennedy 1989). Lo anterior se ve ratificado al observar marcadas inserciones musculares en las crestas iliacas de ambos individuos adultos, que también se han relacionado con el remo (Aspillaga et al. 2006). Aunque la presencia de estas huellas de marcadores ocupacionales no pueden ser atribuidas de forma unívoca a la realización de una actividad particular (Kennedy 1998), creemos que a la luz de toda la información disponible en el sitio (Castro 2011), tanto ecofactual como artefactual, nos parece que existe un sustento material robusto para sostener nuestra hipótesis en un nivel inicial.

Aunque para Llagostera (2005) la navegación representa la etapa final del proceso de maritización y ocurriría en épocas postarcaicas, Núñez (2003) sugiere que las primeras embarcaciones en el norte árido pudieron desarrollarse durante el Arcaico en contextos Chinchorro. Núñez y Contreras (2009) se refieren a una descripción de Capdeville de un "modelo de balsa Chinchorro" encontrado en el cementerio Arcaico de Punta Morada en Taltal. En Copaca 1 encontramos asociaciones de peces de mar abierto como marlín y albacora en contextos arcaicos (Castro et al. 2013), lo que avalaría la hipótesis de Núñez (2003). Recientemente Olguín et al. (2014) sustentan esta misma hipótesis en un área similar y con un rango cronológico análogo.

Se pudo constatar en el miembro inferior de los individuos adultos, facetas de acuclillamiento que puede asociarse a la mantención de una postura forzada acuclillada por períodos prolongados afectando la cara articular astragalina de la tibia, a partir de la hiperflexión de la cadera, tobillo y rodilla (Larsen 1997:185). Además, la presencia de marcadas inserciones musculares en los miembros inferiores indica la realización de una serie de actividades que 
creemos corresponderían a marchas prolongadas y/o constantes, probablemente por terrenos inclinados y/o escarpados, aunque tampoco se puede descartar que estos individuos hayan realizado actividades relacionadas a la natación.

\section{Dieta}

En cuanto al análisis de isótopos, la literatura muestra a individuos asociados a sitios costeros y consumo de dieta marina, que en general no superan un máximo de $\delta 15 \mathrm{~N} 15 \%$, llegando algunos a poco más de $20 \%$. Los altos índices de nitrógeno en los individuos de Copaca 1 estarían evidenciando, al igual que varios sitios del extremo norte de Chile de períodos similares, un consumo marino casi exclusivo (Aufderheide y Kelley 1994; Tieszen et al. 1992; Roberts et al. 2013). La corriente de Humboldt, con su alta diversidad de fauna marina, es la causa de que, al existir una mayor cantidad de seres vivos, las cadenas tróficas son más largas, aumentando el enriquecimiento de nitrógeno (Tieszen y Chapman 1992). En efecto, los isótopos de nitrógeno, al contrario de los de carbono, se van enriqueciendo cerca de $3 \%$ en cada nivel de la cadena alimenticia, sobre todo si provienen del mar. Por ello, mientras más larga sea la cadena trófica marina, los valores de nitrógeno serán más altos (Kline et al. 2013).

Es muy probable que en nuestro caso los altos valores de $\delta 15 \mathrm{~N}$ puedan además "enriquecerse por efecto de un importante estrés fisiológico causado por vivir en ambientes áridos con escasos recursos de agua" (Ambrose y Sikes 1991; Barberena 2002). Estudios en herbívoros en zonas áridas de África en comparación a los que viven en zonas húmedas y bajas temperaturas lo demuestran (Barberena 2002). Este estudio no ha sido corroborado en poblaciones humanas vivas.

El carbono proveniente del colágeno se enriquece por el consumo de plantas $\mathrm{C} 4$, circunstancia improbable para las muestras de Copaca 1, situada en la desértica costa arreica de Antofagasta. Sin embargo, también puede presentar altos índices por mayor consumo de otras proteínas, como las provenientes de una dieta marina (entre otros, Tieszen et al. 1992). En nuestra muestra, cuyos niveles de $\mathrm{N}$ son muy altos, no hay duda que los altos niveles de $\delta 13 \mathrm{C}$ col se deben atribuir a esta dieta y no al maíz, imposible de cultivar en este ambiente hiperárido, y menos en fechas arcaicas.
Los resultados provenientes del carbono de la apatita también permiten confirmar la ingesta de una rica dieta marina. Sin embargo, la apatita de los isótopos estables de carbón indican escasas fuentes alimenticias de lípidos y carbohidratos derivados esencialmente de plantas C3 (Kline et al. 2013; Tabla 2), las que se podrían atribuir al consumo de herbívoros, como el guanaco.

Asimismo, de acuerdo con las cantidades de colágeno proveniente de $13 \mathrm{C}$ y $15 \mathrm{~N}$, los dos individuos masculinos adultos obtuvieron la mayoría de su dieta proteica de peces y mamíferos marinos, en tanto que el individuo femenino joven (Individuo 3), con índices más bajos de nitrógeno, la obtuvo de crustáceos (Kline et al. 2013). A pesar de la escasez de la muestra, si esto se confirma en otras investigaciones, podría indicar una dieta diferenciada por género o edad.

Los hallazgos asociados a los restos humanos en Copaca incluían el consumo casi exclusivo de toda clase de elementos marinos vertebrados e invertebrados: peces, mariscos, mamíferos y aves marinas. Fuera de estos elementos propios de la cadena trófica marina, solo se evidenciaron algunos restos de mamíferos terrestres, esencialmente de camélidos.

\section{Conclusiones}

Considerando lo expuesto, se pueden establecer varios puntos importantes para estudiar. No obstante, debido a lo reducido de la muestra, se debe enfatizar que nuestras conclusiones no pretenden ser resolutivas, sino preliminares y propositivas.

1. Los individuos del sitio Copaca 1 corresponden a parte de una población con un modo de vida de cazadores-recolectores marinos, lo que se puede inferir de los contextos de los basurales exhumados, el alto enriquecimiento de nitrógeno y valores de espaciamiento entre apatita y colágeno en los isótopos estables y de las actividades posibles de ser reconstruidas a partir de huellas paleopatológicas y musculares, además de las mencionadas inferencias sobre su dieta. El alto enriquecimiento de nitrógeno podría deberse a lo discutido en páginas precedentes, junto al hecho de que estos habitantes casi no se movían de la costa que les daba el sustento, a excepción de probables escasos movimientos al interior para cazar guanacos y posiblemente obtener algunos vegetales. Es importante destacar la presencia de enfermedades metabólicas en 


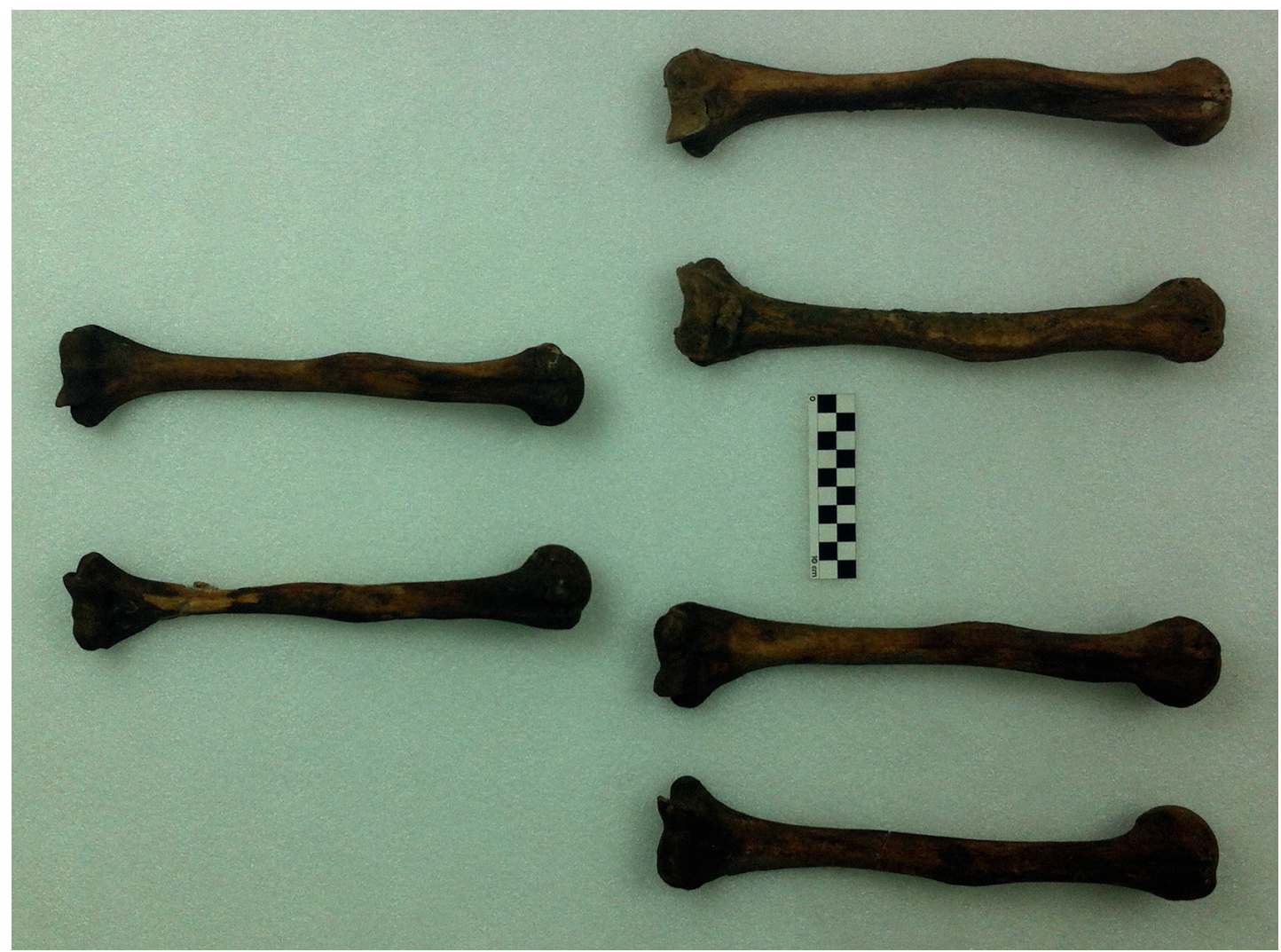

Figura 7. Retroversión humeral en miembros superiores de los Individuos 1 y 2 (derecha) y ausencia del rasgo en el miembro superior del Individuo 3 (izquierda).

Humeral retroversion in upper limbs of Individuals 1 and 2 (right) and absence of the feature in the upper limb of Individual 3 (left).

estos individuos que nos hablaría de sus hábitos alimenticios, específicamente del hecho de consumir peces y mamíferos marinos crudos, además de una deficiencia de consumo de vegetales ricos en vitamina $\mathrm{B} 9$.

2. En este grupo se podrían haber generado diferencias sexuales del trabajo, toda vez que los individuos masculinos estarían realizando actividades extractivas marinas, como el buceo y la navegación, aunque debido al tamaño de nuestra muestra, ello solo se puede establecer como una propuesta para investigar a futuro. Respecto de esto, se considera de gran importancia poder contar con una muestra donde la actividad de la boga sea conocida, con el fin de aumentar el número de variables a analizar, tomando no solo en consideración las variables de marcadores musculares de ocupación, sino que también de enfermedades degenerativas articulares asociadas a ellas (Weiss y Jurmain 2007).

3. En consecuencia, creemos que las evidencias recabadas en otros sectores de la costa arreica del norte de Chile (Núñez y Contreras 2009; Olguín 2014; Urrea 2013), más las aquí presentadas, sugieren que la navegación se habría dado ya en tiempos del período Arcaico y no correspondería exclusivamente a una actividad del período Formativo, como se ha planteado anteriormente (Llagostera 1990).

A modo de cierre, consideramos necesario conocer de manera más extensiva los modos de vida de los grupos humanos que habitaron el sitio Copaca 1, esto requiere contar con una muestra mayor que la ya analizada, para así poder hacer comparable la realidad de este grupo con aquellos encontrados en sitios como Caleta Huelén 42 y cruzar los datos obtenidos de la cultura material, restos humanos y ecofactuales para tener una mirada más completa al modo de vida de los cazadores recolectores marinos de la costa arreica de Antofagasta.

Agradecimientos: A FONDECYT 1100951, quien financió nuestras investigaciones, al equipo 
de investigación que nos acompañó durante el desarrollo del proyecto y al excelente estudio sobre isótopos estables de nuestras muestras, desarrollado por los Dres. Kline, MacKinnon y Eric Bartelink, del Departamento de Antropología de la
Universidad Estatal de California, Chico, que nos ha servido de comparador con los resultados del Centro de Estudios Aplicados de la Universidad de Georgia. A los evaluadores y editores por sus comentarios y dedicación.

\section{Referencias Citadas}

Alfonso, M., V. Standen y V. Castro 2008. The adoption of agriculture among Northern Chile populations in the Azapa Valley, 9000 - 1000 BP. En Ancient Health. Skeletal Indicators of Agricultural and Economic Intensification, editado por M.N. Cohen y G.M.M. Crane-Kramer, pp. 113-129. University of Florida Press, Gainesville.

Alfonso, M., E. Calás y F. Morello 2011. Análisis bioantropológico de un enterratorio humano del Holoceno Tardío en Cabo Nose, Tierra del Fuego, Chile. Magallania 39:147-162.

Allen, L.H. 2008. Causes of vitamin B12 and folate deficiency Food and Nutrition Bulletin 29 (Suppl. 2):S20-S34.

Ambrose, H. y N. Sikes 1991. Soil carbon isotope evidence for Holocene habitat change in the Kenya Rift Valley. Science 253:1402-1405.

Andrade, P., D. Salazar, J. Urrea y V. Castro 2014. Modos de vida de los cazadores-recolectores de la costa arreica del norte grande de Chile: Una aproximación bioarqueológica a las poblaciones prehistóricas de Taltal. Chungara Revista de Antropología Chilena 46:467-491.

Aspillaga, E., M. Castro, M. Rodríguez y C. Ocampo 2006. Paleopatología y estilo de vida: el ejemplo de los Chonos. Magallania 34:77-85.

Aufderheide, A. y M. Kelley 1994. Contributions of chemical dietary reconstruction to the assessment of adaptation by ancient highland immigrants (Alto Ramírez) to coastal conditions an Pisagua; North Chile. Journal of Archaeological Science 21:515-524.

Aufederheide, A. y C. Rodriguez-Martin 1998. The Cambridge Encyclopedia of Human Paleopathology. 1 ra Ed. Cambridge University Press, Cambridge.

Barberena, R. 2002. Los Límites del Mar: Isótopos Estables en Patagonia Meridional. Sociedad Argentina de Antropología, Colección Tesis de Licenciatura, Buenos Aires.

Bass, W. 1987. Human Osteology: A Laboratory and Field Manual. Special Publication No 2. 3ra Ed. Missouri Archaeological Society, Columbia.

Bird, D. y R. Bird 2000. The ethnoarchaeology of juvenile foragers: Shellfishing strategies among Meriam Children. Journal of Anthropological Archaeology 19:461-476.

Bridges, P. 1994. Vertebral arthritis and physical activities in the prehistoric southeastern United States. American Journal of Physical Anthropology 93:83-93.

Brothwell, D. 1981. Digging up Bones: The Excavation, Treatment and Study of Human Skeletal Remains. $3^{\text {ra }}$ Edición. Cornell University Press, Cornell.
Buikstra, J. y D. Ubelaker (eds.) 1994. Standards for the Data Collection from Human Skeletal Remains. Arkansas Archaeological Survey Research Series 44.

Campillo, D. y M.E. Subirá 2004. Antropología Física para Arqueólogos. Ariel Prehistoria, Barcelona.

Castelleti, J. 2007. Patrón de Asentamiento y uso de Recursos a través de la Secuencia Ocupacional Prehispana en la Costa de Taltal. Memoria para optar al grado de Magister en Antropología con mención en Arqueología. Convenio Universidad Católica del Norte-Universidad de Tarapacá, Arica.

Castro, V., C. Aldunate y V. Varela 2010. El Proyecto Cobija... Veinte Años Después. Homenaje a Bente Bittmann. Actas del XVII Congreso Nacional de Arqueología Chilena, Valdivia 2006, Vol. 2, pp. 627-639. Universidad Austral de Valdivia-Imprenta América, Valdivia.

Castro, V. 2011. Cronología y Periodificación en la Costa de Antofagasta y su relación con las Tierras Altas. Manuscrito en posesión de la autora.

Castro, V., C. Aldunate y V. Varela 2012. Paisajes Culturales de Cobija, costa de Antofagasta, Chile. Revista Chilena de Antropología 26:97-128.

Castro, V., C. Aldunate, V. Varela, L. Olguín, P. Andrade, F. García-Albarido, F. Rubio, P. Castro, A. Maldonado y J. Ruz 2015. Nuevos aportes al Arcaico en la costa arreica de Antofagasta, norte de Chile, ms en posesión de los autores.

Chimenos, E. 2004. Perspectiva odontoestomatológica en paleopatología. En Paleopatología. La enfermedad no Escrita, editado por A. Isidro y A. Malgosa, pp. 151-162. Editorial Mason, Barcelona.

Cocilovo, J., H. Varela, M. Costa-Junquiera y S. Quevedo 2005. Los pescadores arcaicos de la desembocadura del río Loa (Norte de Chile): El sitio Caleta Huelén 42. Chungara Revista de Antropología Chilena 37:5-19.

Constantinescu, F. y E. Aspillaga 1991. Paleopatología de la columna vertebral en una muestra de indígenas chonos. Actas del XI Congreso Nacional de Arqueología Chilena (Santiago 1988), Tomo III, pp.237-241, Santiago.

Constanzo, D. 2013. Una Aproximación a la Reconstrucción de los Modos de Vida a partir de las Enfermedades Degenerativas Articulares en la Columna Vertebral en las Poblaciones de la Costa Arreica en el Norte Grande de Chile. Memoria para optar al título de Antropólogo Físico. Departamento de Sociología y Antropología, Universidad de Concepción.

Costa-Junqueira, M.A. 2001. Modalidades de enterramientos humanos arcaicos en el norte de Chile. Chungara Revista de Antropología Chilena 33:55-62. 
Dias, G. y N. Tayles 1997. "Abscess cavity" - a misnomer. International Journal of Osteoarchaeology 7:548-554.

Falabella, F., M.T. Planella, E. Aspillaga, L. Sanhueza y R. Tykot 2007. Dieta en sociedades alfareras de Chile central: aporte de análisis de isótopos estables. Chungara Revista de Antropología Chilena 39:5-27.

Flores, S. 2013. Análisis de Paleopatologías Dentales en la Costa Arreica del Norte de Chile. Estudio Comparativo en Poblaciones Costeras del Período Arcaico. Memoria para optar al título de Antropólogo Físico. Departamento de Sociología y Antropología. Universidad de Concepción, Concepción.

Henderson, C. y A. Cardoso 2013. Special issue entheseal changes and occupation: Technical and theoretical advances and their applications. International Journal of Osteoarchaeology 23:127-134.

Hershkovitz, I., B. Latimer, O. Dutour, L. Jellema, S. WishBaratz, C. Rothschild y B. Rothschild 1997. Why do we fail in aging the skull from the sagittal suture? American Journal of Physical Anthropology 103:393-399.

Hillson, S. 2008. Dental pathology. En Biological Anthropology of the Human Skeleton, editado por M. Katzenberg y S. Saunders, pp. 301-340. Segunda edición. Willey-Liss, Nueva Jersey.

Iscan, M.Y. y S.R. Loth 1986. Estimation of age and determination of sex from sternal rib. En Forensic Osteology: Advances in the Identification of Human Remains, editado por K.J Reichs, pp. 68-89. Springfield, Thomas.

Jurmain, R.D. y L. Kilgore 1995. Skeletal evidence of osteoarthritis: A paleopathological perspective. Annals of Rheumatoid Disease 54:443-450.

Kennedy, K.A.R. 1989. Skeletal markers of occupational stress. En Reconstruction of Life from the Skeleton, editado por M.Y. Iscan y K.A.R Kennedy, pp. 129-160. Alan R. Liss, Inc. New York.

Kennedy, K.A.R. 1998. Markers of occupational stress: Conspectus and prognosis of research. International Journal of Osteoarchaeology 8:305-310.

Kline, S., A. Mac Kinnon y E. Bartelink 2013. Stable isotope analysis and paleodiet of ancient humans from the Atacama Desert of coastal Chile. Dp. of Anthropology, California State University, Chico. Manuscrito en posesión de los autores.

Krenzer, U. 2006. Compendio de Métodos Antropológico Forenses para la Reconstrucción de Perfil Osteo-biológico. Serie de Antropología, CAFCA. Guatemala.

Larsen, C.S. 1995. Biological changes in human populations with agriculture. Annual Reviews of Anthropology 24:185-213.

Larsen, C.S. 1997. Bioarchaeology. Interpreting Behavior from the Human Skeleton. Cambridge University Press, Cambridge.

Lizárraga, R. de 1968 [1605]. Descripción breve de toda la tierra del Perú, Tucumán, Río de la Plata y Chile. B.A.E. Editorial Atlas, Madrid.

Llagostera, A. 1990. La navegación prehispánica en el Norte de Chile: bioindicadores e inferencias teóricas. Chungara Revista de Antropología Chilena 24-25:37-51.

Llagostera, A. 2005. Culturas Costeras Precolombinas en el Norte Chileno: Secuencia y Subsistencia de las Poblaciones
Arcaicas. Biodiversidad Marina: Valoración, Usos y Perspectivas. Ed. Universitaria, Santiago.

Llagostera, A. y V. Llagostera 2010. Enterratorios del sitio arqueológico Los Bronces-1. Comuna de Taltal (Región de Antofagasta). Taltalia 3:7-20.

Llagostera, A. y J. Cruz 2010. Evaluación del recurso arqueológico de la costa del Desierto de Atacama para diversificar los destinos del turismo de intereses especiales de la Región de Antofagasta. Taltalia 3:45-55.

Lovejoy, C.O., R.S. Meindl, T.R. Pryzbeck y R.P. Mensforth 1985. Chronological metamorphosis of the auricular surface of the illium: a new method for the determination of age at death. American Journal of Physical Anthropology 68:15-28.

Loyola, A. y N. Reveco 2011. Bioarqueología de la Infancia y Marcadores Ocupacionales en la Costa Arreica Central en el Norte Grande de Chile. Memoria para optar al título de Antropólogo. Departamento de Sociología y Antropología. Universidad de Concepción, Concepción.

Lukacs, J.R. 1989. Dental paleopathology: methods for reconstructing dietary patterns. En Reconstruction of Life From the Skeleton, editado por M.Y. Iscan y K.A.R. Kennedy, pp. 261-286. Alan R. Liss, Inc. New York.

Mann, R.W. y D.R. Hunt 2005. Photographic Regional Atlas of Bone Disease. A Guide to Pathologic and Normal Variation in the Human Skeleton. $2^{\mathrm{da}}$ Edición. Charles C. Thomas Publisher. Springfield.

Meindl, R.S., C.O. Lovejoy, R.P. Mensforth y R.A. Walker 1985. A revised method of age determination using the os pubis, with a review and test of accuracy of other current methods of pubis symphyseal ageing. American Journal of Physical Anthropology 68:29-45.

Molnar, P. 2006. Tracing prehistoric activities: musculoskeletal stress marker analysis of a Stone-Age Population on the Island of Gotland in the Baltic Sea. American Journal of Physical Anthropology 129:12-23.

Núñez, L., V. Zlatar y P. Núñez 1974. Caleta Huelén 42 una aldea temprana en el Norte de Chile (Nota preliminar). Hombre y Cultura, Tomo II (5):67-103. Centro de investigaciones antropológicas, Universidad de Panamá, Panamá.

Núñez, P. 2003. Vivir el Mar. Instituto de Investigaciones Antropológicas, Universidad de Antofagasta, Chile.

Núñez, P. y R. Contreras 2009. A propósito de una miniatura de balsa en Taltal contemporánea con Chinchorro. Taltalia 2:98-110.

Olguín, L. 2011. Historia de un Conchal: Procesos de Formación y Secuencia Ocupacional del Sitio Arqueológico Agua Dulce, Costa Arreica del Desierto de Atacama, Comuna de Taltal, Región de Antofagasta. Tesis de grado para optar al Título de Arqueólogo. Facultad de Ciencias Sociales, Universidad de Chile, Santiago.

Olguín L., D. Salazar y D. Jackson 2014. Tempranas Evidencias de Navegación y Caza de especies oceánicas en la costa Pacífica de Sudamérica (Taltal 7000 años cal. a.p.) Chungara Revista de Antropología Chilena 46:177-192.

Ortner, D.J. 2003. Identification of Pathological Conditions in Human Skeletal Remains. Academic Press, New York. 
Peña-Villalobos, I., L. Olguín, P. Fibia, V. Castro y M. Sallaberry 2013. Aprovechamiento humano de aves marinas durante el Holoceno medio en el litoral árido del norte de Chile. Revista Chilena de Historia Natural 86:301-313.

Poulson, S.R., S. Kuzminsky, G.R. Scott, V.G. Standen, B. Arriaza, I. Muñoz y L. Dorio. 2013. Paleodiet in northern Chile trough the Holocene: extremely heavy $\delta 15 \mathrm{~N}$ values in dental calculus suggest a guano-derived signature? Journal of Archaeological Science 40:4576-4585.

Quevedo, S. 1998. Biología de una Población Arcaica del Norte Semiárido Chileno. Tesis Doctoral, Facultad de Filosofía y Letras, Universidad de Buenos Aires, Buenos Aires.

Reinhard, K. y O. Urban 2003. Diagnosing ancient diphyllobothriasis from Chinchorro mummies. Memorias do Instituto Oswaldo Cruz 98:191-194.

Redlund-Johnell, I. y M.E. Larsson 1993. Subluxation of the upper thoracic spine in rheumatoid arthritis. Skeletal Radiology 22:105-108.

Roberts, A., D. Pate, B. Petruzzelli, C. Carter, M. Westaway, C. Santoro, J. Swift, T. Maddern, G. Jacobsen, F. Bertuch y F. Rothhammer 2013. Retention of hunter-gatherer economies among maritime foragers from Caleta Vitor, northern Chile, during the late Holocene: evidence from stable carbon and nitrogen isotopic analysis of skeletal remains. Journal of Archaeological Science 40:2360-2372.

Rothschild, B. 2000. Porotic hyperostosis as a manifestation of iron deficiency? Chungara Revista de Antropología Chilena 32:85-87.

Salazar, D., D. Jackson, J. L. Guendon, H. Salinas, D. Morata, V. Figueroa, G. Manríquez y V. Castro 2011. Early evidence (ca. 12,000 BP) for iron oxide mining on the Pacific coast of South America. Current Anthropology 52:463- 475.

Sales, J.R., R.K. Beals y R.A. Hart 2007. Osteoarthritis of the costovertebral joints. The results of resection arthroplasty. Journal of Bone and Joint Surgery (Britain) 89:1336-1339.

Scheuer, L. y S. Black. 2000. Developmental Juvenile Osteology. Academic Press, San Diego.

Sisson, T. y C. Lund 1958. The Influence of Maternal Iron Deficiency on the Newborn. The American Journal of Clinical Nutrition 6:376-385.

Standen, V., B. Arriaza y C. Santoro 1995. Una hipótesis ambiental para un marcador óseo: la exostosis auditiva externa en las poblaciones humanas prehistóricas del desierto del norte de Chile. Chungara Revista de Antropología Chilena 27:99-116.

Standen, V.G., B.T. Arriaza y C.M. Santoro 1997. External auditory exostosis in prehistoric chilean populations: A test of the cold water hipótesis. American Journal of Physical Anthropology 103:119-129.

Stuart-Macadam, P. 1989. Nutritional deficiency diseases: A survey of scurvy, rickets and iron - deficiency anemia. En Reconstruction of Life from the Skeleton, editado por M.Y. Iscan y K.A.R Kennedy, pp. 201-222. Alan L. Riss, Inc. New York.

Stuart-Macadam, P. 2005. Iron deficiency anemia: Exploring the difference. En Sex and Gender in Paleopathological Perspective, editado por A.L. Grauer y P. Stuart - Macadam, pp. 45-63. Cambridge University Press, New York.

Tieszen, L.L. y M. Chapman 1992. Carbon and nitrogen isotopic status of the major marine and terrestrian resources inb the Atacama Desert of Northern Chile. Proceedings of the First World Congress on Mummy Studies Tomo 1, pp. 409-426. Museo Arqueológico y Etnográfico de Tenerife, Cabildo de Tenerife.

Tieszen, L.L., E. Iversen y S. Matzner 1992. Dietary reconstruction based on carbón, nitrogen and sulphur stables isotopes in the Atacama Desert, Northern Chile. Proceedings of the First World Congress on Mummy Studies Tomo 1, pp. 427-441. Museo Arqueológico y Etnográfico de Tenerife, Cabildo de Tenerife.

Urrea, J. 2013. Marcadores Óseos de Actividad en el Miembro Superior de Restos Bioantropológicos del Período Arcaico y Formativo en la Costa Arreica de Chile: Una primera aproximación. Memoria para optar al título de Antropólogo Físico. Departamento de Sociología y Antropología, Universidad de Concepción.

Walker, P.L. 1985. Anemia among Prehistoric Indians of the American Southwest. En Health and Disease in the Prehistoric Southwest, editado por C.F. Merbs y R.J. Miller, pp. 139-162. Arizona State University, Research Papers No 34.

Walker, P.L., R.B. Bathurst, R. Richman, T. Gjerdrum y V.A. Andrushko 2009. The causes of porotic hyperostosis and criba orbitaria: A reappraisal of the Iron deficiency anemia Hypothesis. American Journal of Physical Anthropology 139:109-125.

Weiss, E. y R. Jurmain 2007. Osteoarthritis Revisited: A Contemporary Review of Aetiology. International Journal of Osteoarchaeology 17:437-450. 


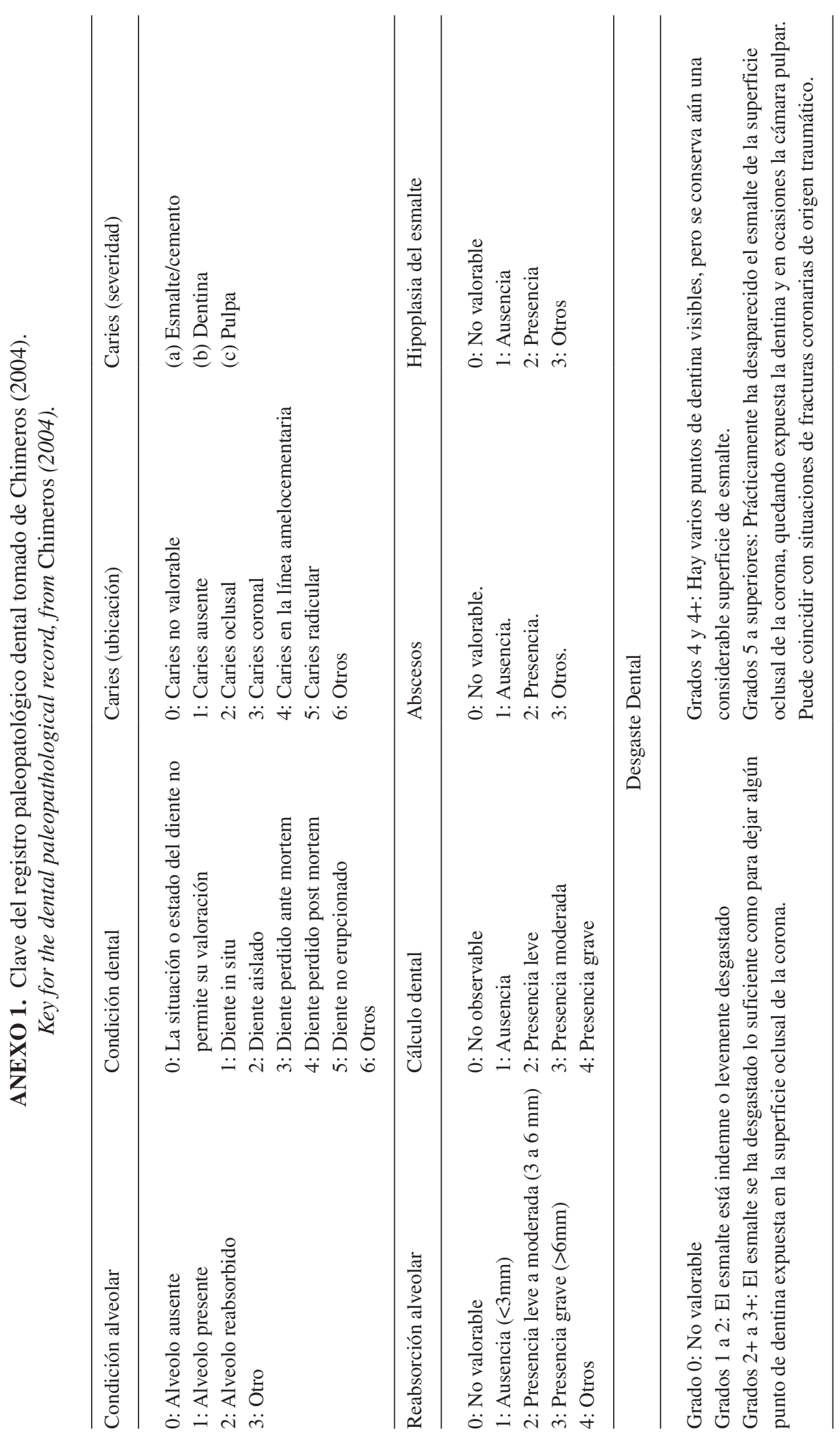

